\title{
$\mathcal{N}=1$ theories, T-duality, and AdS/CFT correspondence
}

\author{
Martin Gremm* \\ Joseph Henry Laboratories, Princeton University, \\ Princeton, NJ 08544, USA \\ E-mail: 'gremm@feynman.princeton. edü
}

\section{Anton Kapustin}

School of Natural Sciences, Institute for Advanced Study Olden Lane,

Princeton, NJ 08540, USA

E-mail: kzapustin@ias.edu

ABstract: We construct an $\mathcal{N}=1$ superconformal field theory using branes of type IIA string theory. The IIA construction is related via T-duality to a IIB configuration with 3-branes in a background generated by two intersecting O7-planes and 7-branes. The IIB background can be viewed as a local piece of an F-theory compactification previously studied by Sen in connection with the Gimon-Polchinski orientifold. We discuss the deformations of the IIA and IIB constructions and describe a new supersymmetric configuration with curving D6-branes. Starting from the IIB description we find the supergravity dual of the large $N$ field theory and discuss the matching of operators and KK states. The matching of non-chiral primaries exhibits some interesting new features. We also discuss a relevant deformation of the field theory under which it flows to a line of strongly coupled $\mathcal{N}=1$ fixed points in the infrared. For these fixed points we find a partial supergravity description.

Keywords: 1'N Expansion, D-branes, Brane Dynamics Gauge Theories, Supersymmetry and Duality.

${ }^{*}$ On leave of absence from MIT, Cambridge, MA 02139, USA 


\section{Contents}

ii. Introduction

2. The IIA construction of the field theory

'2.1' The IIA brane configuration

'2-12 The conformal case: a field theory analysis

!2.3. The non-conformal case

3. The type IIB description

'is.1: T-duality

The seven-brane impurity theory

A supersymmetric IIA configuration with curving six-branes

13. Comparison with F-theory

is. The large $N$ limit

'A. The conformal case

'- The non-conformal case

\section{Introduction}

Brane constructions in string theory provide powerful tools for analyzing field theories in diverse dimensions and with varying amounts of supersymmetry $\left[\begin{array}{l}1 \\ -1\end{array}\right]-2$. For a review

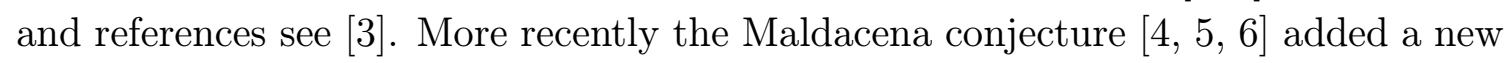
relation between the large $N$ limit of conformal field theories on branes and the near horizon geometry of the corresponding black brane solutions. The original conjecture was stated for $\mathcal{N}=4 \mathrm{SYM}$ realized on $N$ 3-branes, but subsequently more general examples were discovered. One class of such examples are orbifolds of the $\mathcal{N}=4$ configuration [i], is and another includes theories on 3-branes in nontrivial F-theory backgrounds $\left[\begin{array}{l}9 \\ 1\end{array}, 10,1\right]$. All of these constructions give rise to conformal theories with varying amounts of supersymmetry. A third class of theories arises on 3-branes

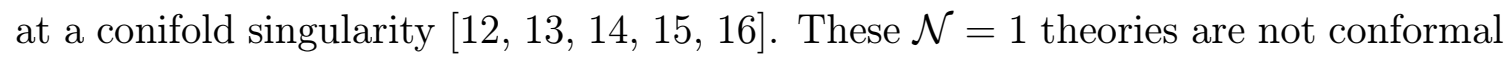
at all scales, but flow to a line of conformal fixed points in the infrared. For all these theories the correspondence between the large $N$ field theory and supergravity was studied in some detail. For branes on a conifold it turned out to be useful to have a type IIA description which is related to the IIB configuration via T-duality [i] 
In this paper, we study a superconformal $\mathcal{N}=1 \operatorname{Sp}(N) \times \operatorname{Sp}(N)$ gauge theory with matter in the fundamental, bifundamental, and antisymmetric representations. We also discuss a specific deformation which preserves $\mathcal{N}=1$ SUSY but breaks conformal invariance. The resulting theory has a running gauge coupling and flows to a line of superconformal fixed points in the infrared. For both of these theories we give a IIA brane construction as well as a IIB orientifold construction. The latter description allows us to obtain the supergravity solution that is dual to the large $N$ limit of the conformal field theory. The type IIA description, on the other hand, provides a simple way to determine the gauge group, the matter content, and the superpotential of the theories in question.

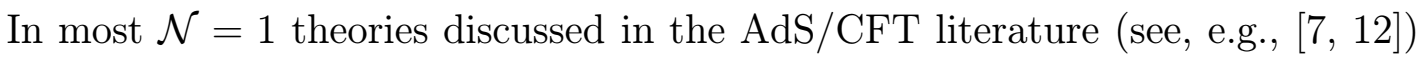
the R-current which is the superpartner of the stress-energy tensor can be fixed uniquely by field theory considerations. For the theories we discuss here this is not the case. There is a one parameter family of candidate R-currents, both in the theory with vanishing beta function, and in its deformation which flows to a line of fixed points. Since the R-charges of the fields are not uniquely determined, there is no field theory prediction for the dimensions of the chiral primary operators. On the other hand, once we have a supergravity dual of the large $N$ field theory, we know which gauge boson on AdS is the superpartner of the graviton. If we are able to match field theory operators with supergravity states, we can determine the R-charges of all fields and therefore the dimensions of all chiral primary operators.

Although there is no firm field-theoretical prediction for the dimensions of fields in the infrared, for the theory with vanishing beta function the most natural assumption is that all fields have canonical dimensions, i.e., that the theory is finite. This will be born out by the supergravity analysis. In the other case, the theory with a running coupling constant, the correct charge assignment in the infrared is harder to guess. Unfortunately the supergravity analysis in this case is on a considerably less solid footing and depends on circumstantial evidence. Nonetheless our analysis suggests a definite R-charge assignment. It would be interesting to find a field theory explanation for it.

The type IIA construction involves D4-branes compactified on a circle as well as NS5-branes, D6-branes, and O6-planes. The gauge theory lives on the D4-branes. Our construction is very similar to the brane configurations that give rise to elliptic

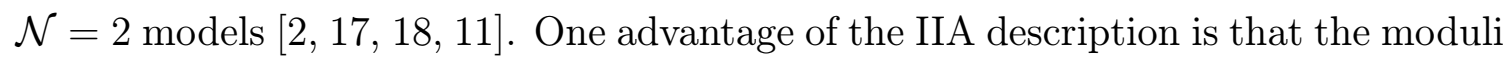
space of the gauge theory is realized geometrically. The flat directions correspond to motions of the 4-branes. Similarly, relevant perturbations of the field theory, such as masses for the matter fields, are also realized geometrically as motions of the 6 -branes. This allows us to identify a 6-brane configuration that gives rise to a superconformal $\mathcal{N}=1$ theory on the 4-branes with an exactly marginal parameter. We can also identify relevant perturbations of the superconformal 4-brane theory that lead to theories with running coupling constants. There is one particular perturbation that 
gives rise to a theory that flows to a line of conformal fixed points in the infrared. The moduli space of the perturbed theory has a Coulomb branch. A generic $\mathcal{N}=1$ theory with a Coulomb branch has a low energy effective gauge coupling that varies over the moduli space. The theory we are considering in this paper has the special feature that the low-energy effective gauge coupling does not depend on the moduli. This will be relevant when we discuss the supergravity description of these theories.

In order to construct the supergravity duals we T-dualize the IIA configuration along the compact direction. This operation turns the D6-branes and the O6-planes into D7-branes and O7-planes. The D4-branes turn into D3-branes probing this

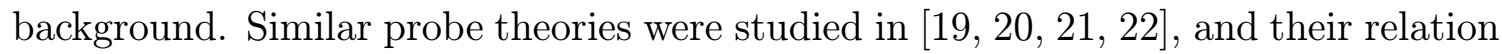

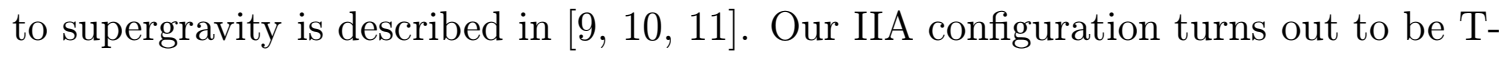

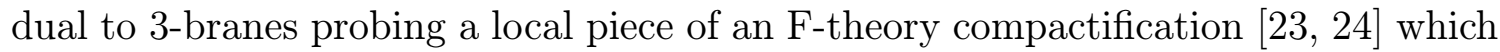
is related to the Gimon-Polchinski model [2] $\left.\overline{5}_{-}\right]$. The simplest such configuration, consisting of two intersecting O7-planes with four coincident 7-branes on top of each, corresponds to the IIA construction of the superconformal 4-brane theory. In the type IIB construction the Ramond-Ramond (RR) charges of the 7-branes are cancelled locally by the charges of the orientifold planes, so the string coupling is constant. Since the type IIB description is a perturbative orientifold, we can find the supergravity dual of the large $N$ limit of the field theory along the lines of $[\overline{9}, 1,10]$. Matching the spectrum of primary operators with the KK modes allows us to determine the $\mathrm{U}(1)_{R}$ charges of all fields in the conformal theory unambiguously. The matching of non-chiral primaries exhibits a new interesting feature: We find a short supergravity multiplet whose field theory counterpart becomes short only when $N \rightarrow \infty$. We interpret this as the evidence that at higher orders in $1 / N$ supersymmetry mixes one-particle and two-particle supergravity states.

It should also be possible to find a supergravity description of the infrared limit of the deformed theory. Although this theory is not conformal, it has a constant lowenergy effective coupling along the Coulomb branch, so the supergravity dual will have a constant dilaton. To find this dual we need to study the deformations of the backgrounds in IIA and IIB and find an explicit map between them. As mentioned before, this is straightforward on the IIA side, since the deformations correspond to motions of the 6-branes. On the IIB side the situation is more involved. We can analyze the deformations on the IIB side by studying the theory on the 7-branes. The eight-dimensional theory on the 7-branes has six-dimensional matter localized at the intersection of orthogonal 7-branes. We analyze the moduli space of this impurity theory following $\left[\overline{2} \overline{6}_{i}\right]$, and find an explicit map between the type IIB and type IIA deformations. Among supersymmetric type IIB deformations there is one that maps to a new IIA brane configuration which involves curving D6-branes in the background of an NS5-brane. The map between deformations also allows us to identify the IIB configuration that gives rise to the non-conformal probe theory with moduli-independent effective coupling. We do not have a complete supergravity 
description of this theory, but a partial description is possible. It supplies enough information to determine the dimension of all chiral operators in the infrared if we use field theory considerations as well.

In section $2 \overline{2}$, we discuss the type IIA construction of the probe theory and list some field theory results that we need in subsequent sections. Section isi-1, contains the T-duality, the analysis of the 7-brane impurity theory, and the map between IIA and IIB deformations. We also briefly discuss the exotic IIA deformation that appears as the counterpart of an ordinary deformation in IIB. In section '出, we analyze the large $N$ limit of our field theories and their supergravity duals. We discuss the matching of operators with Kaluza-Klein modes in the conformal case and present a partial analysis in the non-conformal case.

\section{The IIA construction of the field theory}

\subsection{The IIA brane configuration}

A configuration consisting of D4-branes extending in 01236, D6-branes and O6planes extending in 0123789, and NS5-branes extending in 012389 preserves four supercharges. We obtain an $\mathcal{N}=1$ supersymmetric field theory in four dimensions after compactifying $X_{6}$ on a circle with circumference $2 \pi R_{6}$. Specifically we consider configurations with $N$ D4-branes wrapping the compact $X_{6}$ direction. We put two O6 ${ }^{-}$-planes at $X_{6}=0, \pi R_{6}$ and an NS5-brane and its image at $X_{6}=R_{6} \pi / 2,3 R_{6} \pi / 2$. In order to cancel the total RR charge, we place four physical D6-branes on the circle. An example of such a configuration is shown in figure

These brane configurations are very similar to the configurations that give rise to finite $\mathcal{N}=2$ theories in four dimensions [1] study here can be obtained from one of the $\mathcal{N}=2$ configurations in [i] by rotating the NS5-branes from the 45 directions into the 89 directions. This breaks half of the supersymmetries, giving an $\mathcal{N}=1$ theory in $d=4$.

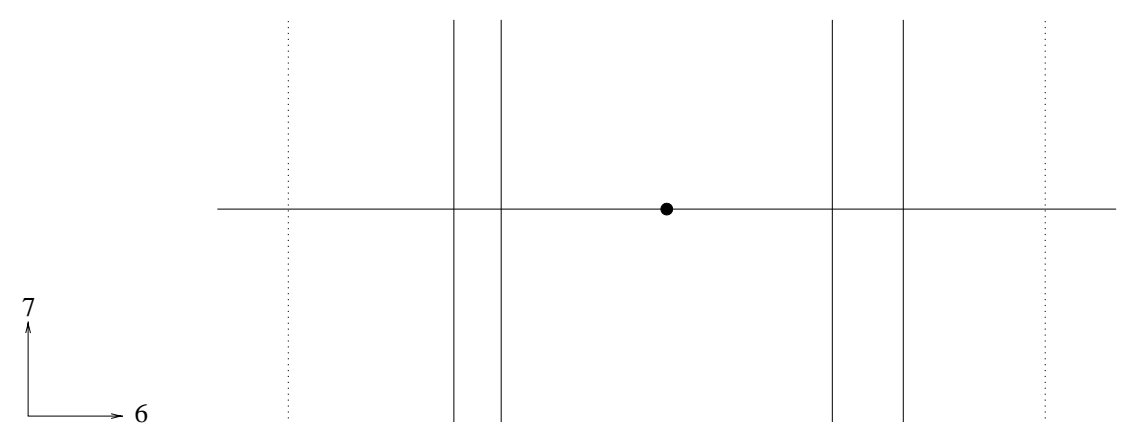

Figure 1: Brane configuration: The vertical dashed lines are the O6-planes, the solid lines are the D6-branes, the horizontal line are the D4-branes, and the point represents the NS5-brane. Only half of the $X_{6}$ circle is shown. 
Using standard techniques [3i], we can determine the matter content and the superpotential of the field theory on the 4 -branes. Unlike the $\mathcal{N}=2$ case, the $X_{6}$ position of the D6-branes will play an important role in our analysis. We need to distinguish two cases that are of interest for the analysis in this paper. Either all 6-branes intersect the NS5-brane, or the four 6-branes are split into two groups of two to the left and right of the NS5-brane (as shown in figure i i). These two choices give rise to physically inequivalent theories. The former configuration yields a line of fixed points (parametrized by the dilaton expectation value) that passes through zero coupling, while the latter corresponds to a non-conformal gauge theory which flows to a line of strongly coupled fixed points.

\subsection{The conformal case: a field theory analysis}

The theory on the 4-branes turns out to be an $\operatorname{Sp}(2 N)_{1} \times \operatorname{Sp}(2 N)_{2}$ gauge theory with matter fields $A_{i}, i=1,2$ in the antisymmetric representation of each of the gauge groups, two bifundamentals $\mathcal{Q}, \tilde{\mathcal{Q}}$, and fundamentals from the 4-6 strings. The brane configuration, and consequently the field theory, admit a symmetry which exchanges the two Sp factors. To determine the number and flavor representations of the fundamentals we need to understand the classical gauge theory on the 6-branes. Note that the worldvolume of the NS5-brane lies within the worldvolume of the D6branes. It was argued in [27] that the 6-branes can break on the NS5-brane (see also $\left.\left[\overline{2} \overline{8}_{1}\right]\right)$. The gauge group on the four 6 -branes turns out to be $\mathrm{U}(4)_{u} \times \mathrm{U}(4)_{d}$, where the two $\mathrm{U}(4)$ factors act on the upper and lower halfs of the 6-branes respectively. One-loop effects break the $\mathrm{U}(4)_{u} \times \mathrm{U}(4)_{d}$ symmetry to $\mathrm{SU}(4)_{u} \times \mathrm{SU}(4)_{d}[2 \overline{9} \overline{1}]$. The matter content of the 6-brane theory includes a bifundamental hypermultiplet from strings connecting upper and lower halfs of the 6-branes. We will have more to say about the 6-brane theory when we discuss the deformations of this background. For our present purposes we only need to know that the gauge group of the 6-brane theory is the flavor group of the probe theory.

The matter content and the superpotential for a 4-brane probe in this background were worked out in [27]. The fundamentals transform as $q=(\square, 1,4,1)$, $\tilde{q}=(\square, 1,1,4), p=(1, \square, \overline{4}, \overline{1})$, and $\tilde{p}=(1, \square, 1, \overline{4})$ under $\operatorname{Sp}(2 N)_{1} \times \operatorname{Sp}(2 N)_{2} \times$ $\mathrm{SU}(4)_{u} \times \mathrm{SU}(4)_{d}$. The superpotential reads

$$
W=h_{1} \tilde{\mathcal{Q}} A_{1} J_{1} \mathcal{Q}-h_{1} \mathcal{Q} A_{2} J_{2} \tilde{\mathcal{Q}}+h_{2} q \mathcal{Q} p+h_{2} \tilde{p} \tilde{\mathcal{Q}} \tilde{q}
$$

Here $J_{1}\left(J_{2}\right)$ is the invariant antisymmetric tensor of $\operatorname{Sp}(2 N)_{1}\left(\operatorname{Sp}(2 N)_{2}\right)$. Following $[\bar{\beta} \overline{0} \overline{0}]$ it is easy to check that this theory has a line of fixed points passing through weak coupling. The one-loop beta function vanishes and the symmetry between the gauge factors implies that both antisymmetric tensors have the same anomalous dimension $\gamma_{A}$, both bifundamentals have $\gamma_{\mathcal{Q}}$ and all fundamentals have $\gamma_{q}$. Therefore, the beta 
functions of the gauge coupling and the Yukawa couplings in the superpotential are

$$
\begin{aligned}
\beta_{g} & \sim 2(N-1) \gamma_{A}+4 N \gamma_{\mathcal{Q}}+8 \gamma_{q}, \\
\beta_{h_{1}} & \sim 2 \gamma_{\mathcal{Q}}+\gamma_{A}, \quad \beta_{h_{2}} \sim \gamma_{\mathcal{Q}}+2 \gamma_{q} .
\end{aligned}
$$

Setting all beta functions to zero gives two independent constraints on the three coupling constants. The remaining coupling constant parametrizes a line of superconformal fixed points. Since setting all anomalous dimensions to zero satisfies the constraints, this line passes through the free point $g=h_{1}=h_{2}=0$. Note that requiring the beta functions to vanish does not fix anomalous dimensions unambiguously. The most natural assumption is that the dimensions of the fields are unchanged as one moves along the fixed line. This would mean that the theory is finite. The supergravity computation in the last section supports this conjecture by showing that this is true in the large $N$ limit.

The moduli space of this theory includes subspaces where it flows to theories with more supersymmetry. For example, giving an expectation value to either $\mathcal{Q}$ or $\tilde{\mathcal{Q}}$ proportional to a unit matrix gives a mass to half of the fundamentals and breaks the gauge group to the diagonal $\operatorname{Sp}(2 N)_{D}$. It is a simple matter to show that the resulting theory flows to an $\mathcal{N}=2$ superconformal theory with gauge group $\operatorname{Sp}(2 N)$, one antisymmetric hypermultiplet, and four hypermultiplets in the fundamental. Giving such expectation values to both $\mathcal{Q}$ and $\tilde{\mathcal{Q}}$ makes all flavors massive and breaks the gauge group to $\mathrm{SU}(N)$. Part of the bifundamentals are eaten by gauge bosons, and the rest give rise to three chiral superfields in the adjoint of $\mathrm{SU}(N)$. This theory flows to $\mathcal{N}=4 \mathrm{SYM}$ in the infrared.

These field theory results are reproduced in the brane construction if we identify the positions of the D4-branes with the field theory moduli in the following way:

$$
\begin{aligned}
& X_{7} \sim \mathcal{Q} \mathcal{Q}^{\dagger}-\tilde{\mathcal{Q}}^{\dagger} \tilde{\mathcal{Q}} \\
& X_{4}+i X_{5} \sim \mathcal{Q} \tilde{\mathcal{Q}}
\end{aligned}
$$

Giving an expectation value to either of the bifundamentals while keeping the other expectation value zero corresponds to moving the 4-branes in the positive or negative $X_{7}$ direction. Turning on both bifundamentals corresponds to moving the 4-branes in the $X_{4}$ and $X_{5}$ directions as well as $X_{7}$. The effect of these motions on the 4-brane theory agrees with the field theory expectations. If we move the 4-branes off the NS5-branes in the $X_{7}$ direction, we can ignore the NS5-brane. The remaining branes preserve eight supercharges, and standard techniques [ij] confirm the matter content and gauge group stated above for the $\mathcal{N}=2$ case. Moving the 4-branes in $X_{4}$ and $X_{5}$ amounts to separating them from all other branes. The theory on the 4-branes is then $\mathcal{N}=4 \mathrm{SYM}$ as expected. 


\subsection{The non-conformal case}

We can deform the background for the 4-brane theory by moving the 6-branes in the $X_{6}$ and $X_{4,5}$ directions. These brane motions are parametrized by expectation values of the two complex scalars, $\mathcal{M}, \tilde{\mathcal{M}}$ in the bifundamental hypermultiplet of the $(1,0)$ theory on the intersection of the 6-branes and the NS5-branes [2]7]. More precisely, we relate the positions of the 6 -branes to $\mathcal{M}, \tilde{\mathcal{M}}$ as follows

$$
\begin{aligned}
& X_{6} \sim \mathcal{M} \mathcal{M}^{\dagger}-\tilde{\mathcal{M}}^{\dagger} \tilde{\mathcal{M}}, \\
& X_{4}+i X_{5} \sim \mathcal{M} \tilde{\mathcal{M}} .
\end{aligned}
$$

To obtain the configuration shown in figure ${ }_{-1}^{1} i_{1}^{\prime}$ we have to set $\mathcal{M}=\operatorname{diag}\left(m_{1}, m_{2}, 0,0\right)$ and $\tilde{\mathcal{M}}=\operatorname{diag}\left(0,0, \tilde{m}_{3}, \tilde{m}_{4}\right)$. These bifundamental expectation values act as mass terms in the 4-brane theory. The corresponding terms in the field theory superpotential are

$$
W=\tilde{\mathcal{M}} q \tilde{q}+\mathcal{M} p \tilde{p}
$$

We will be particularly interested in the case $m_{1}=m_{2}=\tilde{m}_{3}=\tilde{m}_{4}$. In this case the bifundamental expectation values break the $\mathrm{SU}(4)_{u} \times \mathrm{SU}(4)_{d} 6$-brane gauge group to $\mathrm{SU}(2)_{1} \times \mathrm{SU}(2)_{2} \times \mathrm{U}(1)$. After integrating out the massive components of the fundamentals, the superpotential of the 4-brane theory reads

$$
W=h_{1} \tilde{\mathcal{Q}} A_{1} J_{1} \mathcal{Q}-h_{1} \mathcal{Q} A_{2} J_{2} \tilde{\mathcal{Q}}+h_{3} q \mathcal{Q} \tilde{\mathcal{Q}} \tilde{q}+h_{3} \tilde{p} \tilde{\mathcal{Q}} \mathcal{Q} p
$$

The fundamentals now transform as $q=(\square, \mathbf{1}, \mathbf{2}, \mathbf{1}), \tilde{q}=(\square, \mathbf{1}, \mathbf{2}, \mathbf{1}), p=(\mathbf{1}, \square, \mathbf{1}, \mathbf{2})$, and $\tilde{p}=(\mathbf{1}, \square, \mathbf{1}, \mathbf{2})$ under $\mathrm{Sp}(2 N)_{1} \times \mathrm{Sp}(2 N)_{2} \times \mathrm{SU}(2)_{1} \times \mathrm{SU}(2)_{2}$. Actually, the superpotential, eq. $\left(\underline{2} . \overline{6}_{1}\right)$, has an accidental $\mathrm{SO}(4)_{1} \times \mathrm{SO}(4)_{2}$ global symmetry under which $q$ and $\tilde{q}$ transform as a $(\mathbf{4}, \mathbf{1})$ while $p$ and $\tilde{p}$ transform as $(\mathbf{1}, \mathbf{4})$.

An analysis along the lines of 300 shows that this theory also has a line of superconformal fixed points. The beta functions are given by

$$
\begin{aligned}
\beta_{g} & \sim 4+2(N-1) \gamma_{A}+4 N \gamma_{\mathcal{Q}}+4 \gamma_{q}, \\
\beta_{h_{1}} & \sim 2 \gamma_{\mathcal{Q}}+\gamma_{A}, \quad \beta_{h_{3}} \sim 1+\frac{1}{2} \gamma_{\mathcal{Q}}+\gamma_{q} .
\end{aligned}
$$

Demanding that the beta functions vanish, we again find that two out of the three constraints are independent, leaving us with a line of fixed points. In this case, however, the line does not pass through weak coupling, since at least one of the anomalous dimensions must be nonzero. Again the vanishing of the beta functions alone does not determine the values of anomalous dimensions. In the last section we will argue that supergravity considerations allow us to fix this ambiguity for large $N$ and find $\gamma_{A}=\gamma_{Q}=0, \gamma_{q}=-1$.

As in the conformal case we can analyze the RG flows both in field theory and using the brane picture. From the brane construction it is clear that we flow to 
the same $\mathcal{N}=2$ theory as in the conformal case if we move the 4 -branes off the NS5-brane in the positive or negative $X_{7}$ direction. Moving the 4 -branes in $X_{4,5}$ again yields $\mathcal{N}=4 \mathrm{SYM}$. The analysis in the field theory is a little more involved in this case because the one-loop beta function does not vanish. This implies that there will be threshold effects in the matching of the running gauge coupling. On general grounds one would expect the low-energy effective coupling to depend on the size of the bifundamental expectation values in the field theory. However, if we give arbitrary (nonzero) expectation values to $\mathcal{Q}$ and $\tilde{\mathcal{Q}}$, fields get integrated out at a variety of scales. Assuming that the expectation value of $\mathcal{Q}$ is larger than that of $\tilde{\mathcal{Q}}$, the $\operatorname{Sp}(2 N) \times \operatorname{Sp}(2 N)$ gauge group is broken to the diagonal group at a scale set by $\mathcal{Q}$. The diagonal $\operatorname{Sp}(2 N)_{D}$ is broken to $\operatorname{SU}(N)$ at a scale set by $\tilde{\mathcal{Q}}$, and finally the fundamentals are integrated out at scale $h_{3} \mathcal{Q} \mathcal{\mathcal { Q }}$. Matching the gauge couplings at each of these scales we find that the low-energy effective coupling does not depend on the bifundamental expectation values. This is a special feature of this theory that will be important later on.

\section{The type IIB description}

\subsection{T-duality}

In this section, we describe the IIB configuration which is obtained by T-dualizing the IIA brane configuration of section II along $X_{6}$. Since $\partial / \partial X_{6}$ is not a Killing vector, performing this T-duality is not completely trivial. Similar T-dualities on IIA configurations that preserve $\mathcal{N}=2$ supersymmetry on the 4-branes have appeared in the literature $[1,1, i, 1,1]$. In the $\mathcal{N}=2$ case the $\mathrm{T}$-duality maps the two $\mathrm{O}^{-}$-planes and the four D6-branes to an orientifold 7-plane and four D7-branes. The D4-branes become D3-branes probing this background. The NS5-brane and its mirror image turn into a $\mathbb{Z}_{2}$ orbifold acting on the 7-brane coordinates transverse to the D3-brane.

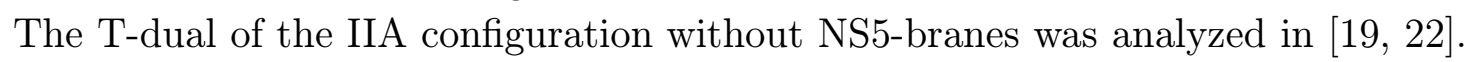

Our configuration differs from the $\mathcal{N}=2$ case by the orientation of the NS5branes. Since this modifies the T-duality considerably we discuss it in some detail here.

Our first goal is to T-dualize the NS5-branes and the pair of $\mathrm{O} 6^{-}$-planes. The other branes can be added later. We begin by separating the NS5-brane and its image in the $X_{4,5}$ directions. The T-dual of the two NS5-branes is a two-center Taub-NUT space. Recall that the two-center Taub-NUT space can be thought of as a circle fibered over $\mathbb{R}^{3}$ so that its radius vanishes at two points on $\mathbb{R}^{3}$ (the centers). In the present case $\mathbb{R}^{3}$ is parametrized by $X_{4}, X_{5}, X_{7}$, while the coordinate along the circle is T-dual to $X_{6}$. The positions of the centers correspond to the positions of the NS5-branes in $X_{4}, X_{5}, X_{7}$. In the IIA configuration the orientifold projection ensures that position of the physical NS5-brane and its image are related by a reflection of 
the $X_{4,5}$ coordinates. The T-dual orientifold projection should therefore impose a similar constraint on the location of the centers of the Taub-NUT. The Taub-NUT metric has the following form

$$
\begin{aligned}
d s^{2}= & \left(\frac{4}{b^{2}}+\frac{1}{R_{+}}+\frac{1}{R_{-}}\right)^{-1}\left[d \sigma+\left(\frac{Z_{+}}{R_{+}}+\frac{Z_{-}}{R_{-}}\right) d \arctan \left(\frac{Y}{X}\right)\right]^{2}+ \\
& +\left(\frac{4}{b^{2}}+\frac{1}{R_{+}}+\frac{1}{R_{-}}\right)\left[d X^{2}+d Y^{2}+d Z^{2}\right]
\end{aligned}
$$

where

$$
Z_{ \pm}=Z \pm Z_{0}, \quad R_{ \pm}^{2}=X^{2}+Y^{2}+Z_{ \pm}^{2}
$$

The $\mathbb{R}^{3}$ base is parametrized by $X, Y, Z$, the two centers are located at $\left(0,0, \pm Z_{0}\right)$, and $\sigma$ is the $4 \pi$-periodic coordinate on the circle fiber. The parameter $b$ is the asymptotic radius of the fiber. The reflection of $X_{4,5}$ in the IIA picture map into reflections of $Z$ and one other coordinate of $\mathbb{R}^{3}$, say $Y$.

We will be interested in the limit when the asymptotic radius of the circle fiber, $b$, becomes infinitely large, while the T-dual circle parametrized by $X_{6}$ shrinks to zero. In this limit the two-center Taub-NUT space becomes an $A_{1}$ ALE space, also known as Eguchi-Hanson space. It is useful to change coordinates [3]1] to transform the metric above into the Eguchi-Hanson form:

$$
\begin{aligned}
X & =\frac{1}{8} \sqrt{r^{4}-a^{4}} \sin (\theta) \cos (\psi), & Z & =\frac{1}{8} r^{2} \cos (\theta), \\
Y & =\frac{1}{8} \sqrt{r^{4}-a^{4}} \sin (\theta) \sin (\psi), & \sigma & =2 \phi,
\end{aligned}
$$

where $a^{2}=8 Z_{0}$ and $\psi$ has period $2 \pi$. The orientifold-induced projection $(Y, Z) \sim$ $(-Y,-Z)$, implies the identification $(\theta, \psi) \sim(\pi-\theta,-\psi)$ for the angular coordinates. The fixed locus of this identification is a two-dimensional submanifold of the EguchiHanson space which has the topology of a cylinder. Next we want to bring the NS5-brane and its image back to the origin of the $X_{4,5}$ plane in the IIA description, which corresponds to setting $a=0$. For $a=0$ the Eguchi-Hanson metric becomes an orbifold metric on $\mathbb{C}^{2} / \mathbb{Z}_{2}$. To make this explicit we can introduce two complex coordinates

$$
z_{1,2}=r \exp \left(\frac{i \phi}{2}\right)\left(\cos \left(\frac{\theta}{2}\right) \exp \left(\frac{i \psi}{2}\right) \pm i \sin \left(\frac{\theta}{2}\right) \exp \left(-\frac{i \psi}{2}\right)\right) .
$$

In these coordinates the $a=0$ Eguchi-Hanson metric becomes flat. The identification $\psi \rightarrow \psi+2 \pi$ requires that we identify $\left(z_{1}, z_{2}\right) \rightarrow\left(-z_{1},-z_{2}\right)$ as expected for $\mathbb{C}^{2} / \mathbb{Z}_{2}$. The additional orientifold identification acts on the new coordinates as $\left(z_{1}, z_{2}\right) \rightarrow\left(z_{1},-z_{2}\right)$, and acting with both orientifold and orbifold identifications flips the sign of $z_{1}$. The orientifold projections have two fixed planes, $z_{1,2}=0$, which we identify with two $\mathrm{O}^{-}$-planes. To summarize, the NS5-brane together with two $\mathrm{O}^{-}{ }^{-}$ planes become, under T-duality, a pair of intersecting $\mathrm{O}^{-}$-planes with six common directions. 
Now let us put in D-branes. The four physical D6-branes in IIA are located at $X_{4}=X_{5}=0$. Under T-duality they become D7-branes wrapping the circle fiber of the Taub-NUT and located at $Y=Z=0$. In other words, they are wrapped on the invariant cylinder of the orientifold projection. Taking the limit $b \rightarrow \infty, a \rightarrow 0$ we find that the invariant cylinder develops a neck and becomes a pair of planes $z_{1}=0$ and $z_{2}=0$ in $\mathbb{C}^{2} / \mathbb{Z}_{2}$. Thus the four physical D7-branes must be located on these planes. Recall that these planes are the $\mathrm{O}^{-}$-planes and therefore have 7 -brane charge -4 . It follows that the 7-brane charge is cancelled between the D7-branes and the orientifold planes, and the IIB dilaton is constant. Finally, T-duality turns the D4-branes into D3-branes extending in 0123. To summarize, the T-dual of the IIA configuration in the limit when the radius of $X_{6}$ goes to zero consists of an $\mathrm{O}^{-}$-plane with four coincident D7-branes in 01236789 , another $\mathrm{O}^{-}$-plane with four coincident D7-branes in 01234589 and 3-branes in 0123. We will refer to the 7-branes extending in 01234589 as $7^{\prime}$-branes. The orientifold group for this configuration is

$$
G=\left\{1,(-1)^{F_{L}} R_{45} \Omega,(-1)^{F_{L}} R_{67} \Omega, R_{4567}\right\}
$$

where $R$ reflects the coordinates indicated and $\Omega$ is the worldsheet parity.

The splitting of the D6-branes into half-D6-branes discussed in [2]7] becomes obvious after T-duality. Indeed, it follows easily from the above formulas that the location of the upper half 6 -branes, $X_{4}=X_{5}=0, X_{7}>0$ in the type IIA configuration maps to the locus $z_{2}=0$ in IIB. Similarly, the lower halfs of the 6 -branes, $X_{4}=X_{5}=0, X_{7}<0$, map to $z_{1}=0$. Thus the upper halfs of D6-branes map to whole D7-branes located at $z_{2}=0$, while the lower halfs map to whole D7-branes at $z_{1}=0$.

To specify the theory on the 7-branes completely we need to make a consistent choice for the action of the orientifolds on the Chan-Paton factors of the 7-7, 7-7', and $7^{\prime}-7^{\prime}$ strings. There are at least two such choices. One gives rise to an $\mathrm{SO}(8) \times \mathrm{SO}(8)$ gauge symmetry $[32 \overline{2}$, and classically the other yields a $\mathrm{U}(4) \times \mathrm{U}(4)$ gauge group on the 7-branes [2033, 2 The second case is related to the Gimon-Polchinski [2] We will be mainly interested in the second orientifold, which we will refer to as the Sen model. Both of these orientifolds were constructed as compact models with a total of four orientifolds and sixteen physical 7-branes of each kind. The 7-brane gauge groups listed here are the parts of the total 7-brane group that are visible to a 3-brane probe near one of the intersections.

The theory on a 3-brane probe in the Sen model background was analyzed in [르르. The gauge group, matter content, and the superpotential are in complete agreement with the theory we discussed in section '2.2.2. Thus we conclude that the IIA configuration with all 6-branes on top of the NS5-brane is T-dual to a local piece of the Sen model $[2 \overline{3}, 24$. As in the IIA description the flat directions of the field theory correspond to motions of the 3-branes in the 7-brane background. Moving the 3-branes 
off the intersection point along either of the O7-planes corresponds to giving en expectation value to one of the bifundamentals $\mathcal{Q}, \tilde{\mathcal{Q}}$, and moving the 3 -branes off both orientifolds gives an expectation value to both $\mathcal{Q}$ and $\tilde{\mathcal{Q}}$. Separating the 3 -branes in the direction which the 7 - and $7^{\prime}$-branes share corresponds to giving expectation values to the antisymmetric tensors $A_{1}, A_{2}$.

It is instructive to study the deformations of the Sen model and compare these to the deformations of the corresponding IIA construction. The IIA construction has the advantage that all deformations of the background correspond to moving the 6-branes or the NS5-branes. In the IIB picture only some of the deformations are geometric, others correspond to Wilson lines. Once the map between IIA and IIB deformations is established, we can also find the IIB description of the second (non-conformal) IIA configuration discussed in section $2 . \overline{3}_{-}^{\prime}$

Sen $[2 \overline{2} \overline{3}, \overline{2} \overline{2} \overline{4}]$ ] has studied the deformations of the compact model in great detail. In the compact case the field theory on the 7 -branes turns out to be a $(1,0)$ theory in six dimensions. Since our IIB configuration is non-compact, we cannot simply use Sen's results. In fact, in our case the theory on the 7-branes is not even six-dimensional, instead it is an eight-dimensional theory with six-dimensional impurities. Such theories

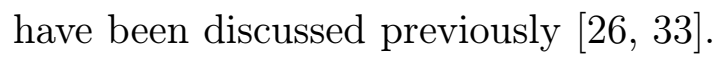

Before we launch into an analysis of the impurity theory we need to discuss the matter content of the 7-brane theory. A single $\mathrm{O}^{-}$-plane with four coincident 7branes gives rise to an $\mathcal{N}=1 \mathrm{SO}(8)$ theory in eight dimensions. The bosonic degrees of freedom in the eight-dimensional vector multiplet consist of a vector field and a complex scalar, both in the adjoint of the gauge group. The second $\mathrm{O}^{-}$-plane in our configuration breaks half of the supersymmetries and imposes projections on fields in the vector multiplet. With the projection matrices for the Sen model $[225$ surviving constant modes of the fields are a vector and a complex scalar in the $\mathbf{6}+\overline{\mathbf{6}}$. These fields account for the 7-7 strings and there are similar fields on the $7^{\prime}$-branes from $7^{\prime}-7^{\prime}$ strings. The $7-7^{\prime}$ strings are localized at the intersection of 7 - and $7^{\prime}$-branes. They yield a single hypermultiplet of the six-dimensional $(1,0)$ theory on the intersection, which transforms as a $(\mathbf{4}, \mathbf{4})$ under the (classical) $\mathrm{U}(4)_{7} \times \mathrm{U}(4)_{7^{\prime}}$ gauge group.

\subsection{The seven-brane impurity theory}

In this section, we analyze the supersymmetric vacua of the impurity theory on the 7-branes and compare them with the vacua of the T-dual IIA configuration. We expect the vacuum field configurations to be translationally invariant in the six directions common to the 7 - and $7^{\prime}$-branes. Focusing now on the 7 -branes, we see that we can capture the physics by studying the dependence of the 7-brane fields on the remaining two directions transverse to the $7^{\prime}$-branes. The $7^{\prime}$-branes and the $07^{\prime}-$ plane intersect this two-dimensional plane in a point. To set up the impurity theory we use a complex affine coordinate $z$ on the plane and define $A_{\bar{z}}=(1 / 2)\left(A_{1}+i A_{2}\right)$, where $A_{i}$ are the two components of the $\mathrm{SO}(8)$ gauge field living on the 7 -branes. 
The 7-brane theory also contains a complex scalar, $\Phi$, in the adjoint of $\mathrm{SO}(8)$ that describes the transverse fluctuations of the 7-branes. The bifundamental $(\mathcal{M}, \tilde{\mathcal{M}})$ from the $7-7^{\prime}$ strings is localized at the point $z=0$. A very similar theory (without orientifold projections) was described in [2]theory is given by the solution of the equations

$$
F_{z \bar{z}}-\left[\Phi, \Phi^{\dagger}\right]=\delta(z)\left(\mathcal{M} \mathcal{M}^{\dagger}-\tilde{\mathcal{M}}^{\dagger} \tilde{\mathcal{M}}\right), \quad \bar{D} \Phi=-\delta(z) \mathcal{M} \tilde{\mathcal{M}}
$$

where $F_{z \bar{z}}=\partial A_{\bar{z}}-\bar{\partial} A_{z}-\left[A_{z}, A_{\bar{z}}\right]$ and $\bar{D}=\bar{\partial}-A_{\bar{z}}$. These equations are known as Hitchin equations with sources. They are analogous to the $D$ and $F$ flatness conditions in ordinary supersymmetric field theories. A similar set of equations describes the impurity theory on the $7^{\prime}$-branes.

To make contact with the notation in [23, 24$]$ we write all 7 -brane fields as antisymmetric $8 \times 8$ matrices with certain constraints on the entries. This reflects the origin of the fields in the impurity theory. Without the O7'-plane, both $A_{\bar{z}}$ and $\Phi$ would transform in the adjoint of $\mathrm{SO}(8)$. Orientifolding with $\mathrm{O}^{\prime}$ puts additional constraints on these fields

$$
\Phi(z)=P \Phi^{T}(-z) P^{-1}, \quad A(z)=P A^{T}(-z) P^{-1}
$$

where

$$
P=\left(\begin{array}{cc}
P_{4} & 0 \\
0 & -P_{4}
\end{array}\right), \quad P_{4}=\left(\begin{array}{cc}
0 & \mathbf{1}_{2 \times 2} \\
-\mathbf{1}_{2 \times 2} & 0
\end{array}\right)
$$

Orientifolding also breaks the gauge group from $\mathrm{SO}(8)$ down to the group of all continuous $\mathrm{SO}(8)$-valued functions satisfying $g(z)=P g(-z) P^{-1}$. In particular, at $z=0$ the gauge group reduces to $\mathrm{U}(4)$. The orientifold projections allow the bifundamentals to be arbitrary complex $8 \times 8$ matrices that commute with $P[2 \overline{2}]$. The impurity equations are consistent if the products of the bifundamentals on the right-hand side of eq. ( $\left(\bar{b} . \overline{6}_{1}^{\prime}\right)$ are antisymmetrized in the gauge indices.

We need to find all, possibly $z$-dependent, field configurations that satisfy the impurity equations, eq. (13.6- $)$, modulo gauge transformations. To this end we make the following ansatz

$$
A_{\bar{z}}=\frac{T}{z}, \quad \Phi(z)=\Phi_{0}+\frac{\Phi_{s}}{z} .
$$

Here $T, \Phi_{0}$, and $\Phi_{s}$ are constant antisymmetric $8 \times 8$ matrices. Imposing the constraints, eq. ( $\left.\underline{\underline{B}} \cdot \overline{Z_{1}}\right)$, determines that $\Phi_{0}$ transforms in the $\mathbf{6}+\overline{\mathbf{6}}$ of $\mathrm{U}(4)$ while $T$ and $\Phi_{s}$ transform as adjoints. The background gauge field, $A_{\bar{z}}$, can be interpreted as a flat connection that gives rise to a Wilson line around the intersection point at $z=0$. The constant part of the scalar field, $\Phi_{0}$, corresponds to the asymptotic (i.e., $z \rightarrow \infty)$ positions of the 7-branes in the directions transverse to the O7-plane, while the singular part, $\Phi_{s}$, parametrizes a deformation of the shape of the 7-branes. 
The moduli space of the impurity equations, eq. (3. rather different physics. The simplest situation arises if all bifundamental expectation

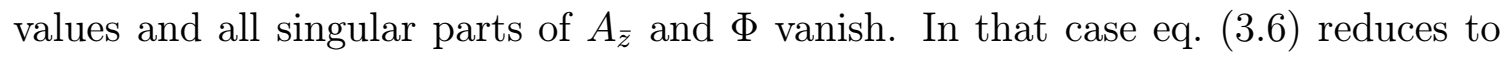
the condition

$$
\left[\Phi_{0}, \Phi_{0}^{\dagger}\right]=0
$$

which is solved by

$$
\Phi_{0}=\left(\begin{array}{cc}
0 & \phi \\
-\phi & 0
\end{array}\right), \quad \phi=\operatorname{diag}\left(\phi_{1}, \phi_{2}, \phi_{1}, \phi_{2}\right) .
$$

As in ref. [23], the two complex parameters, $\phi_{1,2}$, parametrize the transverse position of two pairs of 7-branes. We discuss the corresponding IIA deformation in the next section. For the remainder of this section we set $\Phi_{0}=0$.

The impurity equations, eq. ( $(\overline{3} \cdot \overline{6})$, become inhomogeneous once we turn on an expectation value for the bifundamental fields. Since $\bar{\partial}(1 / z) \sim \delta(z)$, and the righthand side of eq. ( right form to satisfy the impurity equations with nonzero bifundamental expectation values.

The most generic expectation value of the bifundamentals for which the impurity equations have solutions reads

$$
\begin{gathered}
\mathcal{M}=\left(\begin{array}{cc}
M_{1} & 0 \\
0 & M_{2}
\end{array}\right), \\
M_{1}=\left(\begin{array}{cccc}
m_{1} & 0 & -i m_{1} & 0 \\
0 & m_{2} & 0 & -i m_{2} \\
i m_{1} & 0 & m_{1} & 0 \\
0 & i m_{2} & 0 & m_{2}
\end{array}\right), \quad M_{2}=\left(\begin{array}{cccc}
m_{3} & 0 & i m_{3} & 0 \\
0 & m_{4} & 0 & i m_{4} \\
-i m_{3} & 0 & m_{3} & 0 \\
0 & -i m_{4} & 0 & m_{4}
\end{array}\right),
\end{gathered}
$$

and an expectation value of the same form, but with $m_{i}$ replaced by $\tilde{m}_{i}$, for $\tilde{\mathcal{M}}$. The impurity equations determine the expectation values of the other fields in terms of $\mathcal{M}$ and $\tilde{\mathcal{M}}$. The residue of $\Phi$ is given by

$$
\Phi_{s}=\operatorname{diag}\left(\Phi_{1}, \Phi_{2}\right)
$$

where

$$
\Phi_{1}=\left(\begin{array}{cccc}
0 & 0 & -\phi_{1} & 0 \\
0 & 0 & 0 & -\phi_{2} \\
\phi_{1} & 0 & 0 & 0 \\
0 & \phi_{2} & 0 & 0
\end{array}\right), \quad \Phi_{2}=\Phi_{1}\left(\phi_{1} \rightarrow-\phi_{3}, \phi_{2} \rightarrow-\phi_{4}\right),
$$

with $\phi_{i} \sim m_{i} \tilde{m}_{i}$. The matrix $T$ in the gauge connection has the same structure as $\Phi_{s}$, except that $\phi_{i}$ is replaced by $t_{i} \sim\left|m_{i}\right|^{2}-\left|\tilde{m}_{i}\right|^{2}$. 
Before discussing this general solution, we will focus on two special cases. If we set $m_{i}=\tilde{m}_{i}$, the right-hand side of the first impurity equation vanishes and only the residue of $\Phi$ is turned on. This expectation value of the bifundamentals breaks the $\mathrm{U}(4) \times \mathrm{U}(4)$ gauge group to a diagonal subgroup. If all $m_{i}$ are equal this subgroup is $\mathrm{U}(4)_{D}$, and for generic values of $m_{i}$ we find $\mathrm{U}(1)^{4}$. Since the 7 -

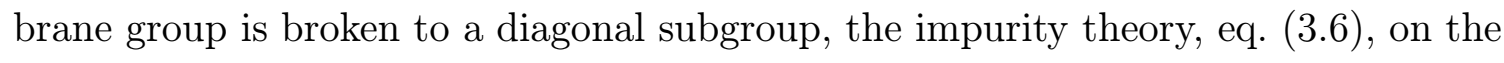
7-branes and the corresponding impurity theory on the $7^{\prime}$-branes contain the same information. Therefore it is sufficient to consider only the 7-brane theory. The field $\Phi(z)$ describes the shape of the 7-branes. For large $z$ the 7-branes asymptote to the O7-plane as in the unperturbed case, while they approach the O7'-plane for small $z$. Thus we conclude that turning on this bifundamental expectation value deforms pairs of intersecting 7 - and $7^{\prime}$-branes into a single smooth 7-brane that interpolates between the 7 - and $7^{\prime}$-branes. This result agrees with the F-theory analysis in [2 24$]$, where this behavior was interpreted as fusing the 7 - and $7^{\prime}$-branes together.

There are also solutions of the impurity equations with nonzero gauge connection and $\Phi_{s}=0$. We find one such solution if we set $m_{1}=m_{2}=\tilde{m}_{3}=\tilde{m}_{4}$, and all other components of the bifundamentals vanish. For this choice the right-hand side of the second equation in eq. ( $\left({ }_{3} . \overline{6}_{1}\right)$ ) vanishes, which implies $\Phi_{s}=0$, and $t_{1}=t_{2} \sim\left|m_{1}\right|^{2}$, $t_{3}=t_{4} \sim-\left|m_{1}\right|^{2}$. This bifundamental expectation value breaks the $\mathrm{U}(4)_{7} \times \mathrm{U}(4)_{7}, 7$ brane gauge group to a diagonally embedded $\mathrm{U}(2) \times \mathrm{U}(2)$. Note that this deformation is purely non-geometric. Since $\Phi(z)=0$, the 7 -branes have the same shape as in the case without any bifundamental expectation values.

It is now a simple matter to identify these two singular solutions with the corresponding deformations in the IIA construction. The first solution with $T=0$, $\Phi_{s} \neq 0$ corresponds to moving the 6 -branes off the NS5-brane in the $X_{4,5}$ direction. If none of the 6-branes coincide, the $\mathrm{U}(4) \times \mathrm{U}(4)$ gauge symmetry on the 6-branes is broken to $\mathrm{U}(1)^{4}$. This is in complete agreement with the impurity analysis. Note that a deformation that corresponds to fusing 7 and $7^{\prime}$-branes together in the IIB description maps into a simple brane motion in the IIA construction, which involves reconnecting the upper and lower halfs of the 6-branes.

The second singular solution with $T \neq 0, \Phi_{s}=0$ also corresponds to a simple brane motion in the IIA description. We identify turning on $m_{1}$ with the motion of two pairs of 6 -branes in the $X_{6}$ direction. The classical gauge group on the 6 -branes is $\mathrm{U}(2) \times \mathrm{U}(2)$ as expected from the IIB analysis. This brane motion also requires that we reconnect the upper and lower halfs of the 6 -branes, so that the resulting 6-brane group is a diagonal subgroup of the original $\mathrm{U}(4) \times \mathrm{U}(4)$ gauge symmetry. This is in perfect agreement with the analysis of the 7-brane impurity theory.

It is straightforward to discuss more general choices for the bifundamental expectation values. The bifundamental expectation values are parametrized by eight complex numbers, $m_{i}$ and $\tilde{m}_{i}$, which determine the matrices $T$ and $\Phi_{s}$ completely. The four parameters in $T$ map into the $X_{6}$ position of the 6 -branes in the IIA descrip- 
tion and the entries in $\Phi_{s}$ correspond to the $X_{4,5}$ positions. Thus we find complete agreement between the brane motions in the IIA description and the moduli corresponding to singular fields in the impurity theory.

\subsection{A supersymmetric IIA configuration with curving six-branes}

The deformations we discussed so far are rather complicated in the IIB picture and correspond to simple brane motions in the IIA description. In fact, all simple brane motions in the IIA description are accounted for. However, there is a very simple brane motion in IIB, namely the constant solution of the impurity equations given in eq. ( $\left(\overline{3} . \overline{1} \overline{1}_{1}\right)$, that should have a counterpart in the IIA description. Since this deformation corresponds to moving pairs of 7-branes off the orientifold, we can find an explicit equation describing the position of these branes. In terms of the coordinates in eq. ( const. Starting from this expression we can reverse the coordinate transformations that took us from the Taub-NUT space to the flat coordinates on $\mathbb{C}^{2} / \mathbb{Z}_{2}$. This provides an expression for the world volume of the 7-brane in the Taub-NUT coordinates. Since the 7-branes wrap the fiber of the Taub-NUT and the fiber T-dualizes to the compact $X_{6}$ direction, it is straightforward to find the equation for the worldvolume of the corresponding 6 -brane. The result is $X_{4}^{2}-c X_{7}-c^{2} / 4=0$, i.e., a parabola in the $X_{4}-X_{7}$ plane. Figure ${ }_{2}^{2}$ shows the IIA configuration which is T-dual to the following IIB situation: All 7'-branes are coincident with the $\mathrm{O}^{\prime}$ '-plane, and one pair of

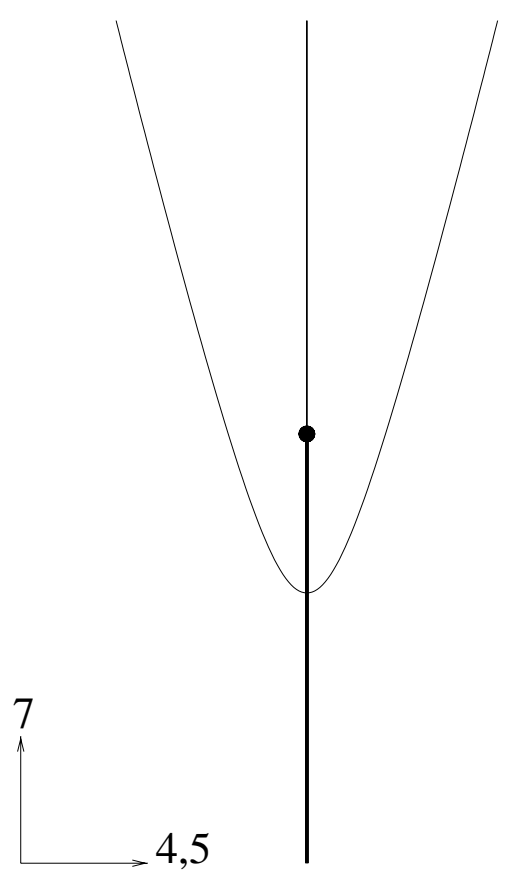

Figure 2: Type IIA configuration for nonzero expectation value of the 6 . The dot represents the NS5-brane, the thick line corresponds to four half 6-branes, the thin line corresponds to two half 6-branes and the curving line is another 6brane.

7-branes is displaced from the O7-plane.

From this picture one can see that turning on the constant complex scalar on the 7-brane corresponds to fusing two upper halfs of the 6-branes together and moving them off the NS5-brane as shown in the figure. On the IIB side it is obvious that this deformation preserves all supersymmetries. This is somewhat harder to see on the IIA side. Presumably the $H$-field produced by the NS5-brane stabilizes the curved worldvolume of the D6-brane.

The effect of this deformation on the probe theory is what we expect from the IIB picture. There we move two 7-branes away from the 3-branes sitting at the intersection point of the orientifold planes. This gives a mass to half of the fundamentals from 7-3 strings. In the IIA picture the deformation accomplishes the same. In the 
IIB picture moving the 3-branes along the $\mathrm{O}^{\prime}$-plane and transverse to the O7-plane corresponds to giving the bifundamental field $\mathcal{Q}$ in the probe theory an expectation value [2]2]. Thus it is possible to move the 3 -branes away from the intersection of the orientifolds towards the intersection of the pair of 7-branes with the ${ }^{7} 7^{\prime}$-plane by giving an expectation value to one of the bifundamentals. This is also reflected in the IIA description. We can move the 4 -branes in the negative $X_{7}$ direction by giving an expectation value to one of the bifundamentals (see section '2.2 2 ). This moves the 4-branes off the NS5-brane and towards the intersection of the lower half-6-branes with the curving 6-brane.

In the IIB description moving a pair of 7-branes away from the O7-plane breaks

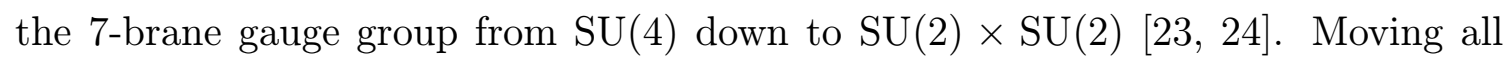
four 7-branes together breaks $\mathrm{SU}(4)$ down to $\mathrm{Sp}(4)$. This implies that the unbroken gauge group on a single curving 6-brane should be $\mathrm{SU}(2)$, while for two coincident curving branes it should be enhanced to $\operatorname{Sp}(4)$. It is not at all clear how to see this from the IIA description.

\subsection{Comparison with F-theory}

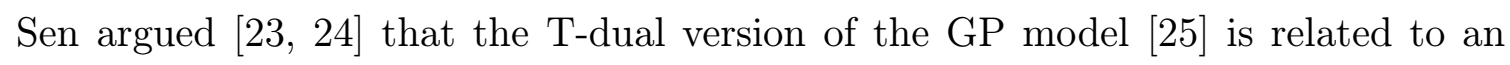
F-theory compactification with certain fluxes through collapsed 2-cycles. The naive candidate for such an F-theory compactification would be a pair of intersecting $D_{4}$ singularities. However, this cannot be directly related to the GP orientifold, since it would give rise to an $\mathrm{SO}(8) \times \mathrm{SO}(8)$ gauge symmetry and contain tensionless strings, while the GP model has $\mathrm{SU}(4) \times \mathrm{SU}(4)$ symmetry and no tensionless strings. The difference is due to NS (and possibly RR) 2-form fluxes through the collapsed 2-cycle at the intersection of the two $D_{4}$ singularities. These fluxes give a mass to 3-branes wrapping this cycle, thereby preventing the appearance of tensionless

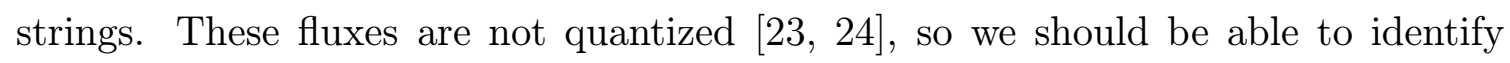
moduli in our IIA description that correspond to turning them off. The NS flux is conventionally identified with the position of the NS5-branes on the $X_{6}$ circle and the RR flux parametrizes the location of the NS5-branes on the M-theory circle. From the IIB point of view, they are both part of a massless hypermultiplet living at the intersection of the $D_{4}$ singularities. In order to turn off the NS flux, we move the NS5-brane and its image as well as all D6-branes to coincide with one of the O6-planes. This configuration has an $\mathrm{SO}(8) \times \mathrm{SO}(8)$ gauge symmetry from the eight upper and eight lower halfs of the 6-branes, as well as tensionless strings from the

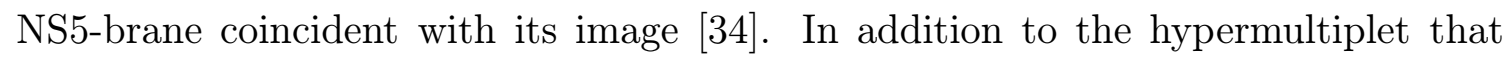
corresponds to moving the NS5-brane off the orientifold in the $X_{4}, X_{5}, X_{6}, X_{10}$ there is now a tensor multiplet whose scalar expectation value corresponds to separating the two NS5-branes in the $X_{7}$ direction. All this agrees with the expectations from F-theory. 


\section{The large $N$ limit}

When the number of D3-branes, $N$, is large there is a dual description of $\mathcal{N}=1$ superconformal theory on the D3-branes in terms of a supergravity on $\mathrm{AdS}_{5} \times X$, where $X$ is an Einstein manifold (or orbifold) This dual description is valid when the t'Hooft gauge coupling, $g_{\mathrm{YM}}^{2} N$, is large. In this section, we will show how the AdS/CFT correspondence works for the conformal gauge theory with $\mathrm{SU}(4) \times$ $\mathrm{SU}(4)$ flavor symmetry discussed in section 2.2, and provide evidence that this theory is finite. We will also provide a partial analysis of the non-conformal theory of section '2.3' in the large $N$ limit and argue that supergravity suggests a definite Rcharge assignment for all the fields in the infrared.

\subsection{The conformal case}

In the conformal case, $X$ is an orientifold of $\mathbf{S}^{5}$. As explained in the previous section, the IIA configuration with $\mathrm{SU}(4) \times \mathrm{SU}(4)$ gauge symmetry on the 6 -branes is T-dual to a local piece of the Sen model. At the $\mathrm{SU}(4) \times \mathrm{SU}(4)$ point, the Sen model is

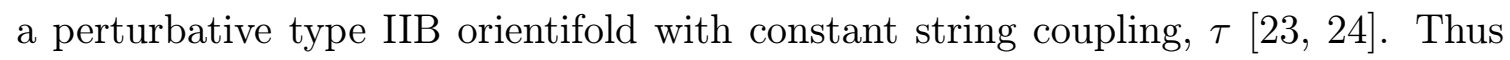
the near-horizon geometry of the 3-branes is obtained by orientifolding $\operatorname{AdS}_{5} \times \mathbf{S}^{5}$.

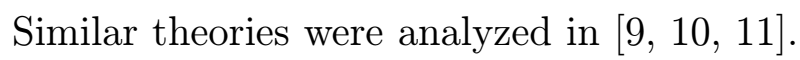

Let us denote the orientifolded five-sphere by $\tilde{\mathbf{S}}^{5}$. The metric on $\tilde{\mathbf{S}}^{5}$ is the angular part of

$$
d s^{2}=\left|d z_{1}\right|^{2}+\left|d z_{2}\right|^{2}+|d w|^{2}
$$

where $w=X_{8}+i X_{9}$ and the variables $z_{1}, z_{2}$ are subject to the identifications $z_{i} \rightarrow$ $-z_{i}$. A U $(1)^{3}$ subgroup of the $\mathrm{SO}(6)$ isometry group of $\mathbf{S}^{5}$ commutes with these identifications. It is convenient to take the generators that rotate $z_{1}, z_{2}$, and $w$ separately as a basis in the Lie algebra of $\mathrm{U}(1)^{3}$. Explicitly, the metric on $\tilde{\mathbf{S}}^{5}$ can be written as

$$
d s_{\tilde{\mathbf{S}}^{5}}^{2}=d \theta_{1}^{2}+\sin ^{2}\left(\theta_{1}\right) d \phi_{1}^{2}+\cos ^{2}\left(\theta_{1}\right)\left(d \theta_{2}^{2}+\sin ^{2}\left(\theta_{2}\right) d \phi_{2}^{2}+\cos ^{2}\left(\theta_{2}\right) d \phi_{3}^{3}\right),
$$

where $\phi_{1,2} \in[0, \pi], \phi_{3} \in[0,2 \pi]$, and $\theta_{1,2} \in[0, \pi]$. The three angles $\phi_{i}$ parametrize rotations in the $z_{1,2}$ and $w$ planes respectively. The periodicity of $\phi_{1,2}$ reflects the identifications on $z_{1,2}$. Since this periodicity of $\phi_{1,2}$ is the only thing which distinguishes $\tilde{\mathbf{S}}^{5}$ from $\mathbf{S}^{5}$, the eigenvalues of the scalar Laplacian on the former can be obtained from those on the latter. The eigenvalue of the scalar Laplacian on $\mathbf{S}^{5}$ is $k(k+4)$, where $k=0,1, \ldots$ In terms of the angular momenta, $m_{i}$, associated with the angles $\phi_{i}$, we have $k=\left|m_{1}\right|+\left|m_{2}\right|+\left|m_{3}\right|+2 l_{1}+2 l_{2}$, where $l_{i}$ are nonnegative integers. The orientifold projection on the bulk supergravity states amounts to keeping modes with even $m_{1}$ and $m_{2}$.

In the $\mathcal{N}=4$ case, the supergravity states with lowest mass squared come from the KK reduction of $h_{a}^{a}$, the dilaton mode of the $\mathbf{S}^{5}$. The AdS masses of these states 


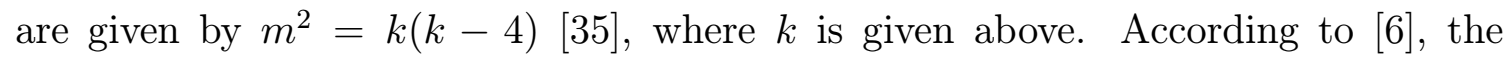
AdS mass of a KK state is related to the dimension of the corresponding boundary operator by $\Delta(\Delta-4)=m^{2}$, which implies $\Delta=k$ for this tower of KK modes. The decomposition of the other supergravity fields yield towers of KK states for which $\Delta=k+n$, where $n$ is a positive integer [6]. We will see below that only for $n=0$ the KK states couple to chiral primary operators. Therefore we will restrict our analysis to the KK modes from the decomposition of $h_{a}^{a}$.

The simplest way to identify chiral primaries is to find all states for which $\Delta=$ $(3 / 2) R$, where $R$ is the $\mathrm{R}$-charge which is part of the superconformal algebra. The Rcurrent is a certain linear combination of the three $\mathrm{U}(1)$ currents. To find this linear combination we first need to determine which supercharges survive the orientifold projection. The orientifold group, $\mathbb{Z}_{2} \times \mathbb{Z}_{2}$, is generated by $\gamma_{1}=R_{z_{2}} \Omega(-1)^{F_{L}}$ and $\gamma_{2}=R_{z_{1} z_{2}}$. Orientifolding by the first generator breaks $\mathrm{SO}(6)$ down to $\mathrm{SU}(2)_{L} \times$ $\mathrm{SU}(2)_{R} \times \mathrm{U}(1)_{N}$ where $\mathrm{U}(1)_{N}$ acts on $z_{2}$ while $\mathrm{SU}(2)_{L} \times \mathrm{SU}(2)_{R}$ acts on $z_{1}, w$. The surviving supercharges $\left(Q_{+}, Q_{-}\right)$transform as $(\mathbf{1}, \mathbf{2})_{1}$ with respect to this group. Orientifolding by $\gamma_{2}$ breaks $\mathrm{SU}(2)_{L} \times \mathrm{SU}(2)_{R}$ down to $\mathrm{U}(1)_{L} \times \mathrm{U}(1)_{R}$. We will denote the sum of the $\mathrm{U}(1)_{L}$ and $\mathrm{U}(1)_{R}$ charges by $\mathrm{U}(1)_{2}$, the difference by $\mathrm{U}(1)_{3}$, and refer to $\mathrm{U}(1)_{N}$ as $\mathrm{U}(1)_{1}$. The charges of $z_{2}, z_{1}$, and $w$ under these three $\mathrm{U}(1)$ 's are given by $(2,0,0),(0,2,0)$, and $(0,0,2)$, respectively. The supercharge $Q_{+}$which survives the second orientifolding has $\mathrm{U}(1)$ charges $(1,1,-1)$. It follows that the $\mathrm{R}$-charge which is in the same superconformal multiplet as the stress-energy tensor is $(1 / 3)\left(2 m_{1}+2 m_{2}-2 m_{3}\right)$. Here $2 m_{1}$ is the $\mathrm{U}(1)_{1}$ charge, $2 m_{2}$ is the $\mathrm{U}(1)_{2}$ charge, and $2 m_{3}$ is the $\mathrm{U}(1)_{3}$ charge. The normalization is chosen so that $Q_{+}$has $\mathrm{R}$-charge 1. It follows that any KK mode with $l_{1}=l_{2}=0, m_{1}, m_{2} \geq 0$ and $m_{3} \leq 0$ should couple to a chiral primary operator in the boundary field theory.

We discussed the identification of geometric motions of 3-branes with flat directions in the 3-brane field theory in the previous section (see also [2-2id). This allows us to determine the $\mathrm{U}(1)$ charges of the fields $A_{1}, A_{2}, \mathcal{Q}, \tilde{\mathcal{Q}}$. The field theory superpotential then fixes the R-charges of the fundamentals $q, \tilde{q}, p, \tilde{p}$. The results are summarized in the table

With these charge assignments in hand it is now a simple matter to match the bulk KK modes and

\begin{tabular}{l|ccc} 
& $\mathrm{U}(1)_{1}$ & $\mathrm{U}(1)_{2}$ & $\mathrm{U}(1)_{3}$ \\
\hline$A_{1,2}$ & 0 & 0 & -2 \\
$\mathcal{Q}$ & 2 & 0 & 0 \\
$\tilde{\mathcal{Q}}$ & 0 & 2 & 0 \\
$\mathrm{q}, \mathrm{p}$ & 0 & 1 & -1 \\
$\tilde{q}, \tilde{p}$ & 1 & 0 & -1
\end{tabular}

Table 1: Charge assignments for the matter fields. the chiral primary operators in the field theory. Let us give some examples. The supergravity spectrum contains a singleton chiral primary with $\mathrm{U}(1)_{3}$ charge -2 and $\Delta=k=1$. This state corresponds to a chiral primary $\operatorname{Tr}\left(A_{1} J_{1}\right)+\operatorname{Tr}\left(A_{2} J_{2}\right)$ in the field theory. ${ }^{1}$ Since $\Delta=1$, this is a free field. For $\Delta=2$ there are three chiral primary states with geometric $\mathrm{U}(1)$ charges $(4,0,0),(0,4,0)$, and $(0,0,-4)$. We

\footnotetext{
${ }^{1}$ The antisymmetric representation of $\operatorname{Sp}(N)$ is reducible and contains a singlet.
} 
identify them with $\operatorname{Tr} \mathcal{Q}^{T} J_{1} \mathcal{Q} J_{2}, \operatorname{Tr} \tilde{\mathcal{Q}}^{T} J_{2} \tilde{\mathcal{Q}} J_{1}$, and $\operatorname{Tr}\left(A_{1} J_{1}\right)^{2}+\operatorname{Tr}\left(A_{2} J_{2}\right)^{2}$. The chiral primary operators with $\Delta=3$ are $\operatorname{Tr}\left[\mathcal{Q} A_{2} \mathcal{Q}^{T} J_{1}+\mathcal{Q}^{T} J_{1} A_{1} J_{1} \mathcal{Q} J_{2}\right], \operatorname{Tr}\left[\tilde{\mathcal{Q}} A_{1} \tilde{\mathcal{Q}}^{T} J_{2}+\right.$ $\left.\tilde{\mathcal{Q}}^{T} J_{2} A_{2} J_{2} \tilde{\mathcal{Q}} J_{1}\right]$, and $\operatorname{Tr}\left(A_{1} J_{1}\right)^{3}+\operatorname{Tr}\left(A_{2} J_{2}\right)^{3}$. They correspond to the KK states with charges $(4,0,-2),(0,4,-2)$, and $(0,0,-6)$ respectively.

The field theory also contains operators that carry charges under the 7-brane

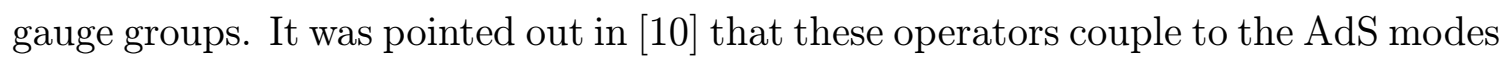
coming from the KK reduction of the 7-brane fields. Our configuration includes an O7-plane with four coincident D7-branes wrapping an $\mathbf{S}^{3}$ defined by $\left|z_{1}\right|^{2}+|w|^{2}=$ const., and similarly an O7'-plane with four D7'-branes wrapped on $\left|z_{2}\right|^{2}+|w|^{2}=$ const. The two 3-spheres intersect over a circle. We can focus on the KK modes from the first $\mathbf{S}^{3}$. These modes couple to operators that are charged under the $\mathrm{SU}(4)_{7}$ subgroup of the $\mathrm{SU}(4)_{7} \times \mathrm{SU}(4)_{7^{\prime}}$ global symmetry group of the probe theory. The modes living on the other $\mathbf{S}^{3}$ couple to similar operators in the field theory that transform under $\mathrm{SU}(4)_{7}$.

The KK reduction of the theory on an O7-plane with four coincident 7-branes was discussed in 10.10. In that case there were twice as many supersymmetries as in ours. The simplest way to compute the KK spectrum in our case is to use the results of [1] 10 ind impose the additional projection from the $\mathrm{O}^{\prime}$-plane.

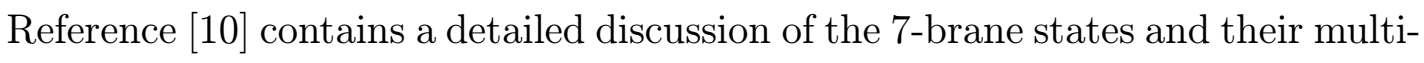
plet structure. The lowest component of the multiplet is a real field in the $(\mathbf{k}, \mathbf{k}+\mathbf{2})_{0}$ representation of $\mathrm{SU}(2)_{L} \times \mathrm{SU}(2)_{R} \times \mathrm{U}(1)_{N}$, where $k=1,2, \ldots$ This mode comes from $\mathrm{KK}$ reduction of the components of the 7-brane gauge field along the $\mathbf{S}^{3}$,

$$
A_{a}=\sum_{k} a_{k} Y_{a}^{k}
$$

where $Y_{a}^{k}$ is the $k$-th vector spherical harmonic on $\mathbf{S}^{3}$. These modes couple to operators of dimension $\Delta=k+1$ in the boundary field theory. For simplicity we will only consider operators with $\Delta=2,3$. The state with $\Delta=2$ transforms in the $(\mathbf{1}, \mathbf{3})_{0}$ and decomposes into modes with $\mathrm{U}(1)^{3}$ quantum numbers $(0,0,0)$ and $(0, \pm 2, \mp 2)$. The $(0,0,0)$ mode has no $\mathrm{U}(1)_{R}$ charge and does not correspond to a chiral primary. The states with $\mathrm{U}(1)^{3}$ charges $(0,2,-2)$ and $(0,-2,2)$ are complex conjugates of each other, so it is sufficient to consider only one of them, e.g., the first. It has R-charge $4 / 3$ and is, therefore, a chiral primary. This state starts out in the adjoint of the $\mathrm{SO}(8)_{7}$ gauge group on the 7-brane. Since it has $m_{2}=1$, it is odd under the additional orientifold projection $\gamma_{2}$. This projection breaks $\mathrm{SO}(8)_{7}$ down to $\mathrm{SU}(4)_{7}$. As explained in $\left[2 \overline{2}_{3}\right.$, , $2 \overline{4}$, states in the adjoint of $\mathrm{SO}(8)_{7}$ which are odd under $\gamma_{1}$ yield $\mathbf{6}+\overline{\mathbf{6}}$ of $\mathrm{SU}(4)_{7}$, while even states give adjoints of $\mathrm{SU}(4)_{7}$. It follows that the $(0,2,-2)$ state yields one complex state in $\mathbf{6}$ and one complex state in $\overline{\mathbf{6}}$. These KK states correspond to operators $q J_{1} q$ and $p J_{2} p$, which transform in the 6 and $\overline{\mathbf{6}}$ of the 7 -brane group respectively. 
The $\Delta=3$ mode is in the $(\mathbf{2}, \mathbf{4})_{0}$ representation and decomposes into even modes with $\mathrm{U}(1)^{3}$ charges $(0,0,-2)$ and $(0,4,-2)$ and their complex conjugates, as well as odd modes with $\mathrm{U}(1)^{3}$ charges $(0,2,0)$ and $(0,2,-4)$ and their complex conjugates. The even $(0,0,-2)$ mode and the odd $(0,2,0)$ mode do not couple to chiral primary operators, because the R-charge does not match the dimension. The even $(0,4,-2)$ mode couples to a chiral primary operator in the adjoint of $\mathrm{SU}(4)$ which we identify as $p J_{2} \tilde{\mathcal{Q}} J_{1} q$. The odd $(0,2,-4)$ mode couples to a chiral primary in the $\mathbf{6}+\overline{\mathbf{6}}$. The corresponding operators are given by $q A_{1} q$ and $p J_{2} A_{2} J_{2} p$.

Other scalars on AdS come from the decomposition of the complex scalar field on the 7-branes. These $\mathrm{KK}$ modes are in the $(\mathbf{k}, \mathbf{k})_{2}$ representation of $\mathrm{SU}(2)_{L} \times$ $\mathrm{SU}(2)_{R} \times \mathrm{U}(1)_{N}$ and couple to operators of dimension $k+2$ [i] $\left.0^{-}\right]$. It is straightforward to decompose and project these modes as we did for the KK modes of the vector field. The $\Delta=3$ case is especially simple, since this mode carries only $\mathrm{U}(1)_{1}$ charge. Since the R-charge and the dimension do not satisfy $\Delta=(3 / 2) R$, this KK mode does not couple to a chiral primary operator. The same is true for the higher KK modes of the complex scalar field.

Finally, there are also states living on the intersection of the 7-branes and $7^{\prime}$ branes which is an $\mathbf{S}^{1}$ embedded in $\mathbf{S}^{5}$. The KK reduction of these states is straightforward, and we will not discuss it.

In the above analysis we have focused on chiral primaries. It is also interesting to ask whether non-chiral states match between field theory and supergravity. Some of the non-chiral scalars we have seen, namely the ones coming from the reduction of complex scalars living on the 7-branes, are descendants of the chiral primaries and therefore match automatically. On the other hand, the non-chiral scalars which come from the KK reduction of the gauge field on the 7 -branes are primary. One may ask whether the superconformal multiplet they live in is long or short.

To answer this question we need to recall some facts about unitary representations of the $\mathcal{N}=1$ superconformal algebra [3] consider multiplets whose primary states have zero spin. Let the $R$ and $\Delta$ be the $\mathrm{R}$-charge and the dimension of the primary. Unitarity puts restrictions on which values of $R$ and $\Delta$ may occur; the allowed possibilities are

(i) $\Delta=R=0$ (the trivial representation),

(ii) $\Delta=(3 / 2)|R|$ (chiral and anti-chiral representations),

(iii) $\Delta \geq(3 / 2)|R|+2$.

Representations of type (iii) with $\Delta>(3 / 2)|R|+2$ contain no null states and therefore are termed long multiplets. Chiral and anti-chiral representations contain null states at level one, i.e., their primaries are annihilated by half of the supercharges. 
These representations are called short. Representations of type (iii) which saturate the inequality are also short; the null states occur at level two. A well-known example of a short multiplet is a linear multiplet which contains a conserved current. It corresponds to the case $R=0, \Delta=2$.

One can check that all non-chiral primaries coming from the reduction of the gauge field on the 7-branes satisfy $\Delta=(3 / 2)|R|+2$ and therefore are in short multiplets of type (iii). In particular, the $(0,0,0)$ mode with $\Delta=2$ we have found above is in fact the lowest component of a linear multiplet. It couples to a field theory operator $q^{\dagger} q-p p^{\dagger}$ in the adjoint of $\mathrm{SU}(4)_{7}$. The corresponding current is simply the $\mathrm{SU}(4)_{7}$ flavor current. The matching of non-chiral primaries with $\Delta=3$ is a bit more involved. The $(0,2,0)$ mode transforms in $\mathbf{6}+\overline{\mathbf{6}}$ of $\mathrm{SU}(4)_{7}$. Its field theory counterparts are $h_{1} p^{\dagger} \tilde{\mathcal{Q}} J_{1} q+h_{2} q J_{1} A_{1}^{\dagger} J_{1} q$ and $h_{1} p J_{2} \tilde{\mathcal{Q}} q^{\dagger}+h_{2} p A_{2}^{\dagger} p$, where the flavor indices are antisymmetrized. The $\mathrm{U}(1)^{3}$ charges of these operators match those of the $(0,2,0)$ mode. To show that these operators live in short multiplets, i.e. are annihilated by $\bar{D}^{2}$, one needs to use the classical equations of motion. The manipulations one has to go through are very similar to those in [37]], and are subject to the same caveats. The use of the classical equations of motion is presumably justified in the weakly coupled regime where $g_{\mathrm{YM}}^{2} N$ is small. The supergravity analysis indicates that the operators in question belong to short multiplets even for large $g_{\mathrm{YM}}^{2} N$. An even more interesting situation arises when one tries to match the non-chiral primary with $\mathrm{U}(1)^{3}$ charges $(0,0,-2)$ and $\Delta=3$. This mode lives in the adjoint of $\mathrm{SU}(4)_{7}$. We claim that it corresponds to the field theory operator $h_{1} q A_{1} J_{1} q^{\dagger}-h_{1} p^{\dagger} A_{2} J_{2} p-h_{2} q \tilde{\mathcal{Q}}^{\dagger} p$. Evaluating the $\bar{D}^{2}$ descendant of this operator using the classical equations of motion, one finds that it does not vanish. Instead, the descendant has the form $(q \tilde{q})(\tilde{p} p)$, i.e. it factorizes into a product of two gauge-invariant operators and is therefore subleading at large $N$. It follows that this field theory operator lives in a long multiplet for finite $N$, but is "close" to being in a short multiplet in the sense that its dimension approaches the unitarity bound as $N \rightarrow \infty$. On the supergravity side this means that the $(0,0,-2)$ one-particle state is in a short multiplet only for $N=\infty$. For finite $N$ the multiplet absorbs another short multiplet made of two-particle states and becomes long.

This concludes our analysis of the AdS/CFT correspondence for the Sen model. There is complete agreement between the spectrum of primary operators in the field theory and the scalar Kaluza-Klein states on AdS as required by the AdS/CFT correspondence [4, $R=(1 / 3)\left(2 m_{1}+2 m_{2}-2 m_{3}\right)$ imply that all chiral fields have canonical dimensions in the infrared. This is the most natural assumption for a theory with vanishing beta function, but as we pointed out in the introduction there is no field theory proof of this. The supergravity computation is only valid for large $N$ and large $g_{\mathrm{YM}}^{2} N$. However, given that for $g_{\mathrm{YM}}^{2} \ll 1$ and $N$ of order 1 the dimensions are also canonical, it appears likely that the theory is finite for all $N$. 


\subsection{The non-conformal case}

Next we discuss the deformed $\mathcal{N}=1$ theory which flows to a line of conformal fixed points in the infrared (section $2.3_{1}$ ). We have already pointed out that although the Wilsonian gauge coupling in this theory depends on the scale, the lowenergy effective gauge coupling does not vary over the moduli space. This implies that the corresponding IIB background should have constant $\tau$. Indeed, in section 'isi-, we showed that the 7-brane background for this configuration is very similar to the background for the conformal theory. As in the conformal case, the 7-branes do not bend and are coincident with the O7-planes. The RR charge of the 7-brane is cancelled locally by the O7-planes, so we expect that the type IIB string coupling is constant. Similarly, the gravitational field of the 7-branes cancels against that of the orientifold planes. Thus it appears that the closed string sector is not affected by this deformation. The only difference between the conformal and the non-conformal case is in the open string sector, namely in the gauge connection on the 7-branes. In the conformal case it is trivial, while in the nonconformal case it is a flat connection which breaks the $\mathrm{SU}(4)_{7} \times \mathrm{SU}(4)_{7^{\prime}}$ group to a diagonally embedded $\mathrm{SU}(2) \times \mathrm{SU}(2)$. To summarize, the deformation of the 7-brane background that leads to the non-conformal theory changes the properties of the theory on the 7-branes, but it appears not to change the closed string sector.

To find a supergravity dual for this non-conformal theory, we need to repeat the analysis above with the new 7-brane background. Since the closed string sector is unchanged, the spectrum of the bulk modes should be the same as before. The matter content of the conformal and the non-conformal theory differ only in the number of flavors and their coupling to the bifundamentals. Therefore both theories have the same spectrum of operators that do not transform under the 7-brane groups. Thus it appears that the dimensions of all chiral primaries uncharged with respect to the flavor group are the same as in the conformal case, i.e., canonical. If antisymmetric tensors and bifundamentals have zero anomalous dimensions then the vanishing of the beta-functions, eq. ( $\left(2, \bar{z}_{1}\right)$, requires that the fundamentals have dimension $1 / 2$. This is actually the lowest dimension for the fundamental allowed by unitarity. To show that this assignment of dimensions, or equivalently of R-charges, agrees with supergravity we would have to show that the KK reduction of the 7-brane theory with the singular flat connection switched on, reproduces the expected dimensions of the chiral primaries that involve the fundamentals. Unfortunately we do not know how to analyze the excitations of the impurity theory around nontrivial vacua, so we cannot check that our solution is consistent. Nevertheless, we get a definite prediction for the infrared dimensions of all fields. It would be interesting to confirm the answer by directly analyzing the perturbative expansion of the non-conformal theory at large $N$. 


\section{Acknowledgments}

It is a pleasure to thank O. Aharony, E. Gimon, J. Maldacena, G. Moore, E. Katz, and E. Witten for helpful discussions. M.G. would like to thank the Institute for Advanced Study for hospitality while this work was in progress. The work of M.G was supported in part by DOE grants \#DF-FC02-94ER40818 and \#DE-FC02-91ER40671, while that of A.K. by DOE grant \#DE-FG02-90ER40542.

\section{References}

[1] A. Hanany and E. Witten, Type IIB superstrings, BPS monopoles, and three-

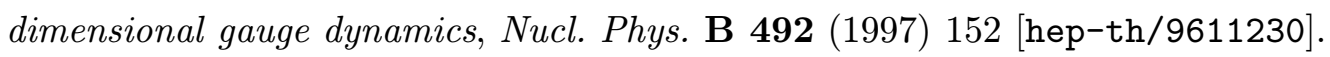

[2] E. Witten, Solutions of four-dimensional field theories via $M$ theory, 'N !

[3] A. Giveon and D. Kutasov, Brane dynamics and gauge theory, thep-th/9802067.'.

[4] J. Maldacena, The large- $N$ limit of superconformal field theories and supergravity,

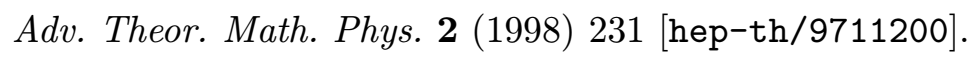

[5] S.S. Gubser, I.R. Klebanov and A.M. Polyakov, Gauge theory correlators from noncritical string theory, 'P

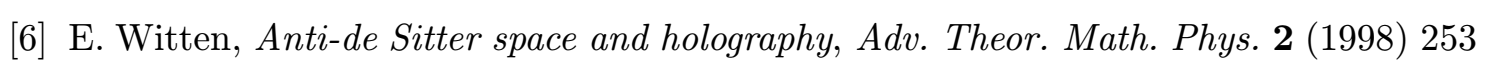
[hep-th/9802150i].

[7] S. Kachru and E. Silverstein, 4d conformal theories and strings on orbifolds, 'Phys.'

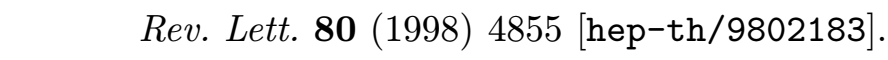

[8] Y. Oz and J. Terning, Orbifolds of $A d S_{5} \times S^{5}$ and $4 d$ conformal field theories, №nucle: :

[9] A. Fayyazuddin and M. Spalinski, Large- $N$ superconformal gauge theories and supergravity orientifolds, iNucl. Phys. B $\mathbf{5 3 5}$ (1998) 219 ; [hep-th/9805096].

[10] O. Aharony, A. Fayyazuddin and J. Maldacena, The Large $N$ limit of $N=2, N=$

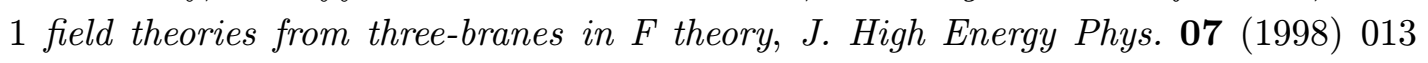
[hep-th/9806159'.

[11] S. Gukov and A. Kapustin, New $N=2$ superconformal field theories from $M / F$ theory orbifolds, iNucl. Phys. B $\mathbf{5 4 5}(1999) 283$ [hep-th/9808175].

[12] I.R. Klebanov and E. Witten, Superconformal field theory on three-branes at a CalabiYau singularity, 'Nucl.

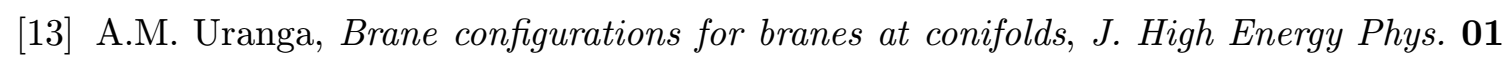
: 
[14] S. Gubser, N. Nekrasov and S. Shatashvili, Generalized conifolds and 4-dimensional $N=1$ superconformal field theory, 'J [hep-th/9811230i].

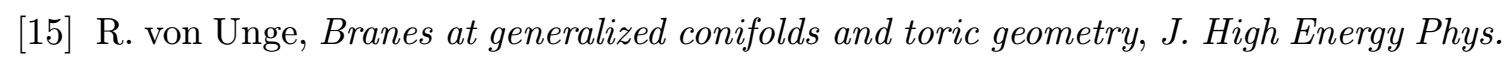
(1) 02 (1999) 023 ihep-th/9901091i.

[16] J. Erlich, A. Hanany and A. Naqvi, Marginal deformations from branes, í. :

[17] A.M. Uranga, Towards mass deformed $N=4 \mathrm{SO}(n)$ and $\mathrm{Sp}(k)$ gauge theories from brane configurations, №cl. Phys. B 526 $(1998) 241$ [hep-th/9803054'.

[18] J. Park and A.M. Uranga, A note on superconformal $N=2$ theories and orientifolds,

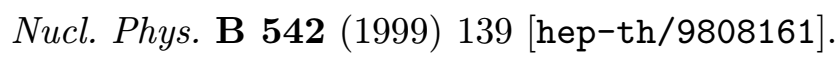

[19] T. Banks, M.R. Douglas and N. Seiberg, Probing F theory with branes, 'Phys. Lett. B. (1)

[20] M.R. Douglas, D.A. Lowe and J.H. Schwarz, Probing F theory with multiple branes,

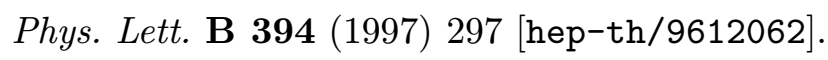

[21] O. Aharony, S. Kachru and E. Silverstein, New $N=1$ superconformal field theories in four-dimensions from D-brane probes,

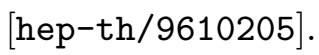

[22] O. Aharony, J. Sonnenschein, S. Yankielowicz and S. Theisen, Field theory questions for string theory answers, iNucl. Phys. B 493 (1997) 177, [hep-th/9611222'].

[23] A. Sen, A nonperturbative description of the Gimon-Polchinski orientifold, 'Nㅡㅁㅣ. : - .

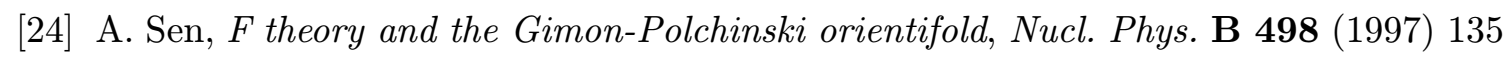
[hep-th/9702061,

[25] E.G. Gimon and J. Polchinski, Consistency conditions for orientifolds and Dmanifolds, 'Phys. Rev. D 54 (1996)_1667', [hep-th/9601038'].

[26] A. Kapustin and S. Sethi, The Higgs branch of impurity theories, iAdv. Theor. Math.'

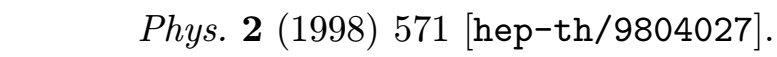

[27] I. Brunner, A. Hanany, A. Karch and D. Lust, Brane dynamics and chiral-non-chiral transitions, Nucl. Phys. B $\mathbf{5 2 8}$ (1998) 197i [hep-th/9801017i].

[28] S. Forste, D. Ghoshal and S. Panda, An orientifold of the solitonic five-brane,

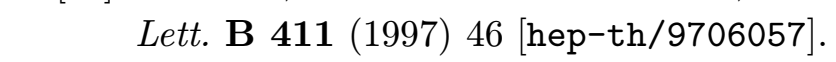

[29] M. Berkooz et al., Anomalies, dualities, and topology of $D=6 N=1$ superstring vacua, iNucl. Phys. B 475 $(1996)$ 115i [hep-th/9605184i]. 
[30] R.G. Leigh and M.J. Strassler, Exactly marginal operators and duality in four-

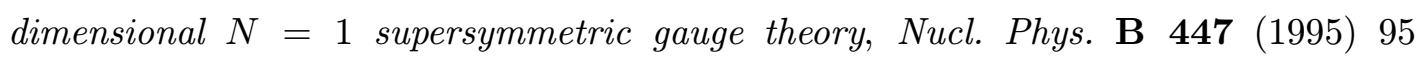
[hep-th/9503121].

[31] M.K. Prasad, Equivalence of Eguchi-Hanson metric to two-center Gibbons-Hawking

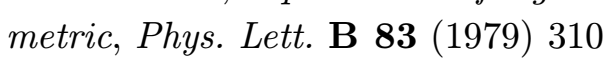

[32] J.D. Blum and A. Zaffaroni, An orientifold from F theory,

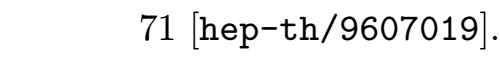

[33] A. Kapustin, $D_{n}$ quivers from branes, iJ. High Energy Phys. 12 $1998 \overline{0}$ [hep-th/9806238i'.

[34] A. Hanany and A. Zaffaroni, Branes and six-dimensional supersymmetric theories, iNucl. Phys B 529 (1998) 180 [hep-th/9712145].

[35] H.J. Kim, L.J. Romans and P. van Nieuwenhuizen, The mass spectrum of chiral $N=$ $2 D=10$ supergravity on $S^{* *} 5$, 'P $\bar{p} \bar{y}$ s.

[36] V.K. Dobrev and V.B. Petkova, All positive energy unitary irreducible representations

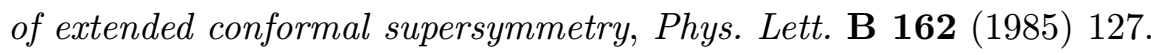

[37] M. Berkooz, A comment on non-chiral operators in SQCD and its dual, 'N- Nucl. Phys.' : 